\title{
Real-space observation of far- and near- field-induced photolysis of molecular oxygen on an Ag(110) surface by visible light
}

Cite as: J. Chem. Phys. 151, 144705 (2019); https://doi.org/10.1063/1.5112158

Submitted: 03 June 2019. Accepted: 08 August 2019. Published Online: 10 October 2019

Chenfang Lin (D), Kei Ikeda, Yoshihito Shiota, Kazunari Yoshizawa, and Takashi Kumagai (D)

\section{COLLECTIONS}

Paper published as part of the special topic on Emerging Directions in Plasmonics

Note: The paper is part of the JCP Special Topic on Emerging Directions in Plasmonics.
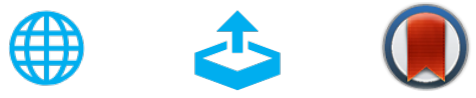

\section{ARTICLES YOU MAY BE INTERESTED IN}

Van der Waals heterostructures of blue phosphorene and scandium-based MXenes monolayers

Journal of Applied Physics 126, 143101 (2019); https://doi.org/10.1063/1.5114850

Ultrafast acoustic phonon scattering in $\mathrm{CH}_{3} \mathrm{NH}_{3} \mathrm{~Pb} \mathrm{P}_{3}$ revealed by femtosecond four-wave mixing

The Journal of Chemical Physics 151, 144702 (2019); https://doi.org/10.1063/1.5120385

Stimulated spin noise in an activated crystal

Journal of Applied Physics 126, 143901 (2019); https://doi.org/10.1063/1.5116901

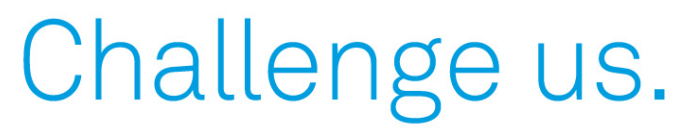

What are your needs for periodic signal detection?
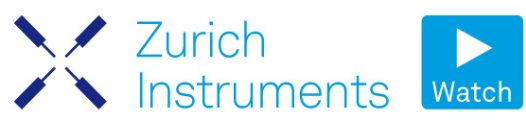

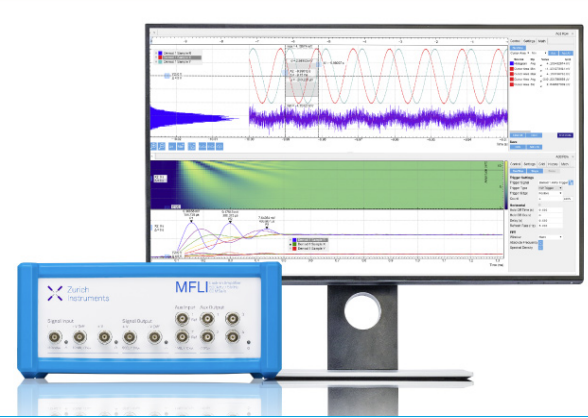

151, 144705 


\title{
Real-space observation of far- and near-field-induced photolysis of molecular oxygen on an Ag(110) surface by visible light
}

\author{
Cite as: J. Chem. Phys. 151, 144705 (2019); doi: 10.1063/1.5112158 \\ Submitted: 3 June 2019 - Accepted: 8 August 2019 • \\ Published Online: 10 October 2019
}

Chenfang Lin, ' (D) Kei Ikeda, ${ }^{2}$ Yoshihito Shiota, ${ }^{2}$ Kazunari Yoshizawa, $^{2}$ and Takashi Kumagai ${ }^{1,3, a)}$ (D)

\begin{abstract}
AFFILIATIONS
${ }^{1}$ Department of Physical Chemistry, Fritz-Haber Institute of the Max-Planck Society, Faradayweg 4-6, 14195 Berlin, Germany

${ }^{2}$ Institute for Materials Chemistry and Engineering, Kyushu University, 744 Moto-oka, Fukuoka, 819-0395 Japan

3JST-PRESTO, 4-1-8 Honcho, Kawaguchi, Saitama 332-0012, Japan
\end{abstract}

Note: The paper is part of the JCP Special Topic on Emerging Directions in Plasmonics.

a) Author to whom correspondence should be addressed: kuma@fhi-berlin.mpg.de

\begin{abstract}
Dissociation of molecular oxygen is an important elementary process in heterogeneous catalysis. Here, we report on a real-space observation of oxygen photolysis on the $\operatorname{Ag}(110)$ surface at $78 \mathrm{~K}$ by far- and near-field excitation in the ultraviolet-near-infrared range using a low-temperature scanning tunneling microscope (STM) combined with wavelength-tunable laser excitation. The photolysis of isolated oxygen molecules on the surface occurs even by visible light with the cross section of $\sim 10^{-19} \mathrm{~cm}^{2}$. Time-dependent density functional theory calculations reveal optical absorption of the hybridized $\mathrm{O}_{2}-\mathrm{Ag}(110)$ complex in the visible and the near-infrared range which is associated with the oxygen photolysis. We suggest that the photolysis mechanism involves a direct charge transfer process. We also demonstrate that the photolysis can be largely enhanced in plasmonic STM junctions, and the cross section is estimated to be $\sim 10^{-17} \mathrm{~cm}^{-2}$ in the visible and the near-infrared range, which appears to be an interesting feature of plasmon-induced reactions from the perspective of photochemical conversion with the aid of solar energy.
\end{abstract}

Published under license by AIP Publishing. https://doi.org/10.1063/1.5112158

\section{INTRODUCTION}

Dissociation of molecular oxygen $\left(\mathrm{O}_{2}\right)$ is a critical step in heterogeneous oxidation processes. Because of its large activation barrier on noble metal catalysts, $\mathrm{O}_{2}$ dissociation often limits the rate of partial oxidation reactions and requires a high operation temperature which causes a number of problems in the energy efficiency and the long-term stability of catalysts. Photochemistry has a potential to attain more selective and moderate material conversion than a thermal process. However, it is a challenging task to induce photochemical reactions by visible-near-infrared light at a low intensity in order to facilitate use of sunlight.

Photochemistry on solid surfaces is of particular importance because a majority of chemical conversions in industry are performed using heterogeneous catalysts. On metal surfaces, despite possible rapid quenching of electronic excitation of adsorbed molecules, it has been demonstrated that surface photochemistry can readily occur. ${ }^{1-4}$ Photo-induced $\mathrm{O}_{2}$ dissociation has been investigated on several metal surfaces, but the reaction cross section remains at $\sim 10^{-21}$ to $10^{-19} \mathrm{~cm}^{2}$ in the UV range. ${ }^{5-8}$ Recently, it has been found that $\mathrm{Au}$ and $\mathrm{Ag}$ nanoparticles can achieve visible-lightdriven reactions under low-power irradiation, ${ }^{9-11}$ reigniting interest in surface photochemistry. Such "plasmonic catalysts" potentially lead to new chemical conversion pathways that cannot be accessed neither by a thermal process nor by conventional surface photochemistry. Elucidation of the underlying physical mechanisms is necessary in order to precisely control the performance of plasmonic catalysis.

Photochemical reactions on metal substrates are rationalized by excitation of charge carriers within an adsorbate-surface system, which is conventionally classified into three possible microscopic mechanisms, namely, direct intra-adsorbate excitation, direct 
charge transfer, and indirect process. The direct intra-adsorbate excitation mechanism assumes the electron transition from occupied to unoccupied states within an adsorbate. The direct charge transfer mechanism assumes the electron transition between the states in the metal substrate and the adsorbate. The indirect mechanism assumes the following process: Hot carriers are first generated within the metal substrate, yielding a nonthermal electron (hole) distribution. Subsequently, the hot carriers scatter through the states (orbitals) centered in the adsorbate and eventually lead to reactions. Similar mechanisms have been invoked to rationalize the microscopic process in plasmonic catalysis and enhanced photocatalytic effects are attributed to excitation of localized surface plasmon resonance (LSPR) of metallic nanoparticles, which can enhance the above three processes. ${ }^{13-16}$ However, the elementary processes in plasmonic catalysis are even more complex than those in conventional surface photochemistry. The efficiency of plasmon-mediated reactions is largely affected by the geometry of metallic nanostructures, and the process in nanoscale cavities plays a crucial role due to the dramatic field enhancement. However, it is very difficult to directly observe plasmon-induced reactions in such nanocavities.

Recently, a low-temperature scanning tunneling microscope (STM) combined with laser excitation has been employed to directly observe plasmon-induced reactions, providing microscopic insights into the underlying mechanism at the single-molecule level. ${ }^{15,16}$ These studies offer a unique opportunity to study surface photochemistry in real space. Here, we report on the direct observation of far- and near-field induced photolysis of $\mathrm{O}_{2}$ on the $\mathrm{Ag}(110)$ surface in the UV-near-infrared range. Silver catalysts are important in the chemical industry such as ethylene epoxidation and methanol oxidation. ${ }^{17,18}$ Among the low index surfaces, $\mathrm{Ag}(110)$ is of particular interest because of the most reactive nature toward $\mathrm{O}_{2}$ activation. $\mathrm{O}_{2}$ photolysis on $\mathrm{Ag}(110)$ was investigated in 1990s, and the reaction was explained by the indirect mechanism. ${ }^{8}$ However, visible-light photolysis was not investigated in detail.

\section{METHODS}

All experiments were performed in an ultra-high vacuum chamber, equipped with a low-temperature STM (Scienta Omicron). All measurements were carried out at $78 \mathrm{~K}$, and the STM images were acquired in the constant current mode. The bias voltage was applied to the sample (denoted as $V_{s}$ ). The $\operatorname{Ag}(110)$ surface was cleaned by repeated cycles of argon ion sputtering and annealing to $700-800 \mathrm{~K} . \mathrm{O}_{2}$ was dosed via a tube doser onto the surface held at $78 \mathrm{~K}$, yielding chemisorbed molecules. Both Au and Ag STM tips were made from polycrystalline wires with electrochemical etching. The Au tip was further milled by focused ion beam (FIB) using a FEI Helios NanoLab G3 FIB-SEM DualBeam system, as described in Ref. 19. For illumination, we used a wavelength tunable laser (NKT Photonics) with the spectral bandwidth of 6-8 $\mathrm{nm}$ in the nearinfrared range and $10 \mathrm{~nm}$ in the visible range. The maximum power used was a few milliwatts. In order to avoid systematic errors caused by misalignment of the relative position between the STM and the beam spot, the lasers were shaped into a $2-3 \mathrm{~mm}$ top-hat square before coupling them to the STM junction (see the supplementary material in Ref. 20 for details).

To investigate the electronic structure of $\mathrm{O}_{2}$ adsorbed on the $\operatorname{Ag}(110)$ surface, we first employed density functional theory
(DFT) calculations with the periodic boundary condition using the PBE functional, ${ }^{21}$ as implemented in the Vienna $a b$ initio simulation package (VASP). ${ }^{22,23}$ We used the projector-augmented wave $(\mathrm{PAW})^{24}$ pseudopotential and the planewave basis set with a cutoff of $500 \mathrm{eV}$. We set the force convergence for the geometry optimization and the threshold for the self-consistent field tolerance to be $1 \times 10^{-2} \mathrm{eV} / \AA$ and $10^{-5} \mathrm{eV}$, respectively. The Brillouin zone with four and nine $k$ points for geometry optimizations and calculations of density of states (DOS) was generated according to the Monkhorst-Pack scheme. ${ }^{26}$ To make a slab model representation of the Ag surface, we used a $(3 \times 4)$ supercell of six layers with $15 \AA$ vacuum along the $z$ direction (see the supplementary material). The lower two layers were kept fixed during geometry optimization.

To identify the optical absorption properties for the obtained geometries in the slab model, we performed the time-dependent DFT (TD-DFT) calculations with the resolution-of-identity approximation $^{26}$ and Tamm-Dancoff approximation ${ }^{27}$ using the PBE functional as implicated in the TURBOMOLE program package. ${ }^{28} \mathrm{We}$ used the def2-SVP basis set ${ }^{29}$ for all atoms and effective core potential for Ag atoms. We considered a $(3 \times 4)$ supercell of three layers. Graphics of all of the obtained structure using DFT and TD-DFT were produced using VESTA. ${ }^{30}$ To analyze the electronic distribution of the excited state, the electron density difference map is visualized using the GaussSum 3.0 program. ${ }^{31}$

\section{RESULTS AND DISCUSSION}

We first discuss the far-field photolysis of $\mathrm{O}_{2}$ on the $\mathrm{Ag}(110)$ surface. The adsorption of $\mathrm{O}_{2}$ at $60-180 \mathrm{~K}$ leads to the molecularly chemisorbed states. ${ }^{32}$ Previous studies reveal that $\mathrm{O}_{2}$ adsorbs on the hollow site with two different orientations: the molecular axis along the [001] and [110] direction. ${ }^{33,34}$ Both species appear as an oval-shaped depression in the STM image with an apparent depth of $\sim 40$ pm [Fig. 1(a)]. However, it is hard to distinguish these two species in a normal STM image unless a CO-modified tip is used for the unambiguous identification. ${ }^{33}$ In addition, according to the previous STM studies, when the $\mathrm{Ag}(110)$ surface is exposed to an $\mathrm{O}_{2}$ gas at $78 \mathrm{~K}$, the fractional population of the [001]- and [110] $-\mathrm{O}_{2}$ species is expected to be $\sim 0.40$ and $\sim 0.54$, respectively, and a small fraction $(<0.06)$ of $\mathrm{O}_{2}$ molecules are dissociatively adsorbed.

After taking an STM image at a relatively large terrace $\left(\sim 50 \times 50 \mathrm{~nm}^{2}\right)$, the surface was illuminated with the tip retracted $\sim 6 \mu \mathrm{m}$ away from the surface to avoid any influence from the presence of the tip. Figure 1(b) shows the STM image obtained after illumination at $520 \mathrm{~nm}$ [the same area with Fig. 1(a)] with an incident laser intensity of $12 \mathrm{~mW} \mathrm{~cm}^{-2}$ on the sample, which equals $\sim 27 \%$ of the sunlight intensity in the visible range. After illumination for $120 \mathrm{~s}$, shallower depressions with an apparent depth of $\sim 23 \mathrm{pm}$ were observed, which are assigned to atomic O. ${ }^{33-35}$ In addition, a rodlike feature with an apparent depth of $\sim 30 \mathrm{pm}$ oriented in the [110] direction also appeared [indicated by the red circle in Fig. 1(b)], which is assigned to next-nearest-neighboring $\mathrm{O}$ atoms. ${ }^{33}$ The STMinduced single $\mathrm{O}_{2}$ dissociation experiment at $\sim 4 \mathrm{~K}$ revealed that the dissociated oxygen atoms adsorb on the hollow $\left(\mathrm{O}_{\mathrm{h}}\right)$ or short-bridge site $\left(\mathrm{O}_{\mathrm{sb}}\right)$, whereas $\mathrm{O}_{\mathrm{sb}}$ is not generated by thermal dissociation at $\sim 170 \mathrm{~K}^{33}$ Therefore, $\mathrm{O}_{\mathrm{h}}$ is assigned to the thermodynamically stable species. ${ }^{33}$ At $\sim 78 \mathrm{~K}$, only $\mathrm{O}_{\mathrm{h}}$ was found after photolysis. The 


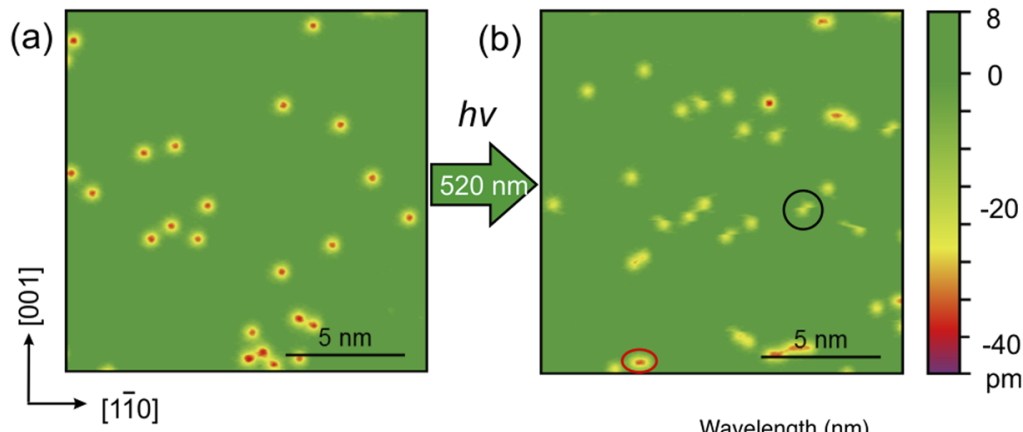

FIG. 1. Far-field $\mathrm{O}_{2}$ photolysis on the $\mathrm{Ag}(110)$ surface. [(a) and (b)] STM images of $\mathrm{O}_{2}$ on $\mathrm{Ag}(110)$ observed at $78 \mathrm{~K}$ $\left(V_{\mathrm{s}}=100 \mathrm{mV}\right.$ and $\left.I_{\mathrm{t}}=20 \mathrm{pA}\right)$, (a) before and (b) after illumination with p-polarized light at $520 \mathrm{~nm}\left(1.6 \times 10^{19}\right.$ photons $\mathrm{cm}^{-2}$ ). The tip was retracted by $\sim 6 \mu \mathrm{m}$ away from the surface during illumination. Scan direction is horizontal. (c) Fraction of unreacted $\mathrm{O}_{2}\left(\mathrm{~N}_{\mathrm{O}_{2}} / \mathrm{N}_{\mathrm{i}}\right)$, atomic $\mathrm{O}\left(\mathrm{N}_{\mathrm{O}} / \mathrm{N}_{\mathrm{i}}\right)$, and total $O\left(\frac{2 N_{\mathrm{O}_{2}}+N_{0}}{N_{\mathrm{i}}}\right)$ as a function of photon fluence at 520

(c)

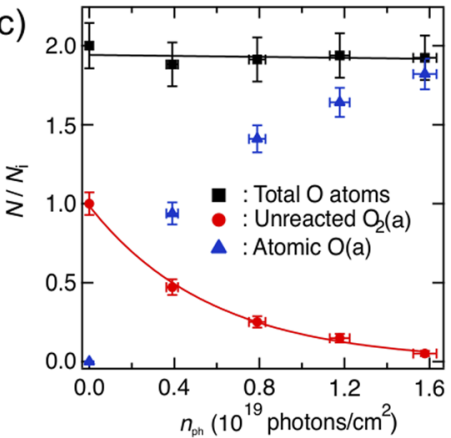

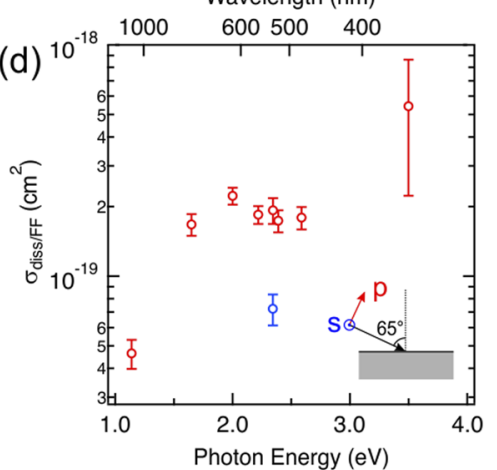
$\mathrm{nm}$ with a flux of $3.3 \times 10^{16}$ photons $\mathrm{cm}^{-2} \mathrm{~s}^{-1}$. Solid curves are the best fitted result of the data points to Eq. (1). (d) Incident photon energy (wavelength) dependence of the farfield photolysis cross section $\sigma_{\text {diss/FF }}$ with p-polarized light (red circles) and a cross section with s-polarized light (blue circle). The geometry of the incident beam and the surface is illustrated in the lower right. photo-dissociated $\mathrm{O}$ atoms diffuse along the [110] direction [indicated by the black circle in Fig. 1(b)].

In order to discuss the $\mathrm{O}_{2}$ photolysis in a quantitative manner, we analyzed the reaction cross section. In Fig. 1(c), the fraction of $\mathrm{O}_{2}$ (unreacted) and atomic oxygen (reacted) is plotted as a function of photon fluence $\left(n_{\mathrm{ph}}\right)$. The fraction represents the number of $\mathrm{O}_{2}$ $\left(N_{\mathrm{O}_{2}}\right), \mathrm{O}\left(N_{\mathrm{O}}\right)$, or total $\mathrm{O}$ atoms $\left(N_{\text {tot }}\right)$ divided by initial $\mathrm{O}_{2}$ numbers $\left(N_{\mathrm{i}}\right)$ counted in a $50 \times 50 \mathrm{~nm}^{2}$ scan area. The standard error resulting from the finite sampling number is given by $\sqrt{N}$ according to a Poisson distribution. Fluence (photons per $\mathrm{cm}^{2}$ ) equals illumination time multiplied by a fixed flux at $3.3 \times 10^{16}$ photons $\mathrm{cm}^{-2} \mathrm{~s}^{-1}$.

After illumination, $91 \%$ of $\mathrm{O}_{2}$ was dissociated. The fraction of the total $\mathrm{O}$ atoms decreases by $<5 \%$. We found that the photolysis follows a first-order rate law and the photolysis rate constant shows a linear dependence on light intensity, indicating that the reaction occurs via a single-photon process (Fig. S1 of the supplementary material). Therefore, we use a cross section that is a measure of the rate constant at a unit photon flux, in this paper as a characterization for the $\mathrm{O}_{2}$ photolysis. The cross section is determined by

$$
N_{\mathrm{O}_{2}}=N_{\mathrm{i}} \exp \left(-\sigma_{\text {diss } / \mathrm{FF}} n_{\mathrm{ph}}\right),
$$

where $\sigma_{\text {diss/FF }}$ is the photolysis cross section by far-field excitation and $n_{\mathrm{ph}}$ is the photon fluence. The photolysis cross section is obtained by fitting the data in Fig. 1(c) to Eq. (1): $\sigma_{\text {diss } / \mathrm{FF}}=(1.7 \pm 0.2) \times 10^{-19} \mathrm{~cm}^{2}$ at $520 \mathrm{~nm}$. Provided the $\mathrm{O}_{2}$ desorption also follows Eq. (1), its cross section can also be estimated by fitting the data of the total $\mathrm{O}$ atoms, yielding the upper limit to be $\sim 10^{-21} \mathrm{~cm}^{2}$.
The $\mathrm{O}_{2}$ photolysis was examined at different wavelengths ranging from 355 to $1090 \mathrm{~nm}$. Figure 1(d) shows the wavelength dependence of $\sigma_{\mathrm{diss} / \mathrm{FF}}$. In the near-infrared and the visible range, $\sigma_{\mathrm{diss} / \mathrm{FF}}$ rapidly increases around $1.5 \mathrm{eV}$ and once saturates at $2 \times 10^{-19} \mathrm{~cm}^{2}$. Then, $\sigma_{\text {diss } / F F}$ further increases in the UV range $(\sim 3.5 \mathrm{eV})$, which can be attributed to the photo-absorption by the $d$-band of the Ag substrate, resulting in efficient hot-carrier generation that eventually leads to the $\mathrm{O}_{2}$ dissociation through the indirect mechanism. ${ }^{8}$ It should be noted that $\sigma_{\text {diss/FF }}$ in the visible range is much higher than that reported previously. ${ }^{8}$ This discrepancy may be explained by different coverage of $\mathrm{O}_{2}$. The coverage in the present case is about $0.01 \mathrm{ML}$ which is much smaller than the saturation coverage $(0.25 \mathrm{ML})$ used in the previous study. ${ }^{8}$ At the saturation coverage, dissociation of $\mathrm{O}_{2}$ must kick out another molecule to yield the adsorption site for the generated $\mathrm{O}$ atoms. This would cause a much lower reaction rate. In addition, the adsorption structure is different at the saturation coverage at which the molecules align with an azimuthal direction parallel to the [001] direction and are tilted away from the surface, ${ }^{32}$ which might also affect the photolysis mechanism. Figure 1(d) also shows the incident-light-polarization dependence at $530 \mathrm{~nm}$. The cross section with s-polarization is smaller than that with p-polarization.

In order to gain insight into $\mathrm{O}_{2}$ photolysis in the visible and near-infrared range, we simulated the adsorption structure and analyzed the optical absorption of the $\mathrm{O}_{2} / \mathrm{Ag}(110)$ complex using TDDFT calculations with a cluster model. Figures 2(a) and 2(b) show the optimized structures of [110]- and [001]- $\mathrm{O}_{2}$ superimposed with the electron density differential map. We confirmed that the slab and cluster model have the same electronic structure and the cluster size 

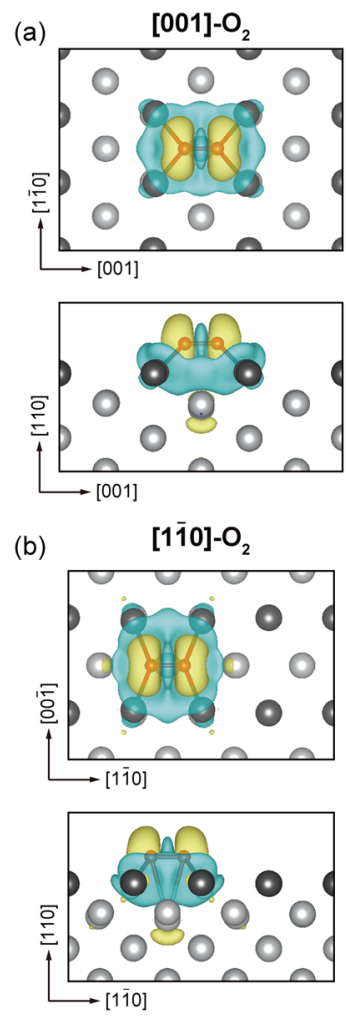

(d) $1000800 \quad 600 \quad 500 \quad 400$

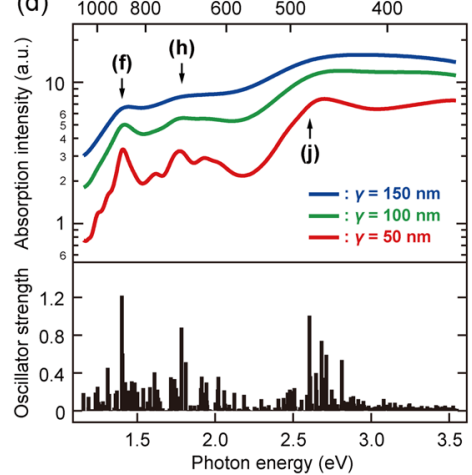

(e)

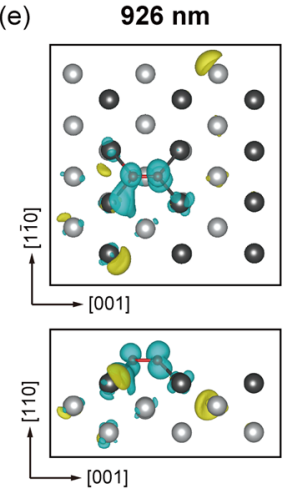

(g)

(h)

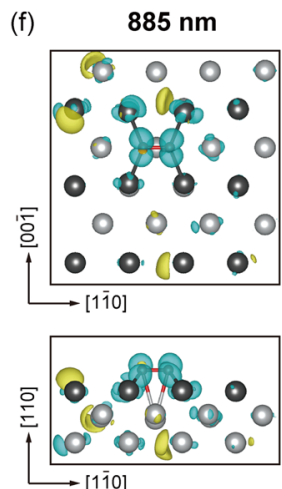

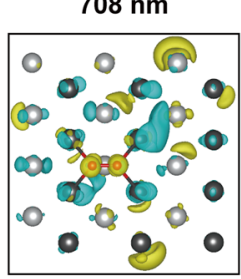

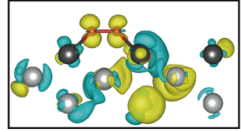

(i)
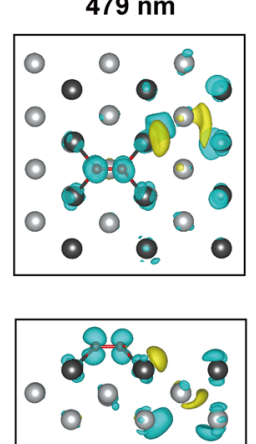

(j)
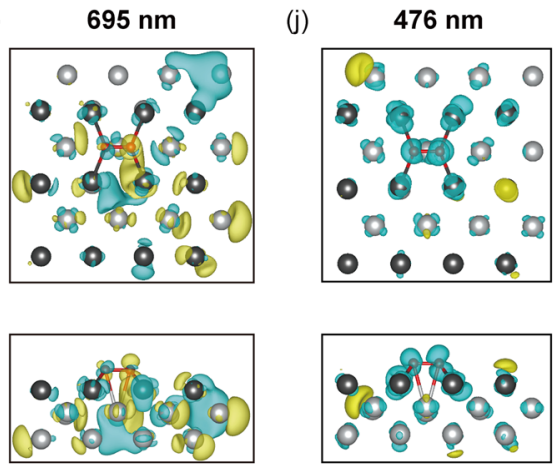

FIG. 2. Simulated structure and optical absorption. [(a) and (b)] Calculated structure of the [001]- and [110]- $\mathrm{O}_{2}$ on the $\mathrm{Ag}(110)$ surface. Red, black, and gray balls indicate $\mathrm{O}$, $\mathrm{Ag}$ atoms at the top, and inner layer, respectively. Isosurfaces of the electron density difference maps $\left[\rho\left(\mathrm{Slab}+\mathrm{O}_{2}\right)-\rho(\mathrm{Slab})-\rho\left(\mathrm{O}_{2}\right)\right.$, where $\rho$ is the electron density] for the adsorbed $\mathrm{O}_{2}$ moiety in the ground state is superimposed. Yellow (blue) indicates an increase (decrease) in the electron density. [(c) and (d)] The simulated absorption spectra (colored curves) of the [001]- and [1 10$]-\mathrm{O}_{2}$, respectively. The oscillator strength (black bars) is broadened by a Lorentzian function with the width $(\gamma)$ of $50,100,150 \mathrm{~nm}$. $[(e)-(j)]$ Changes in the electron density upon electronic transition. The yellow (blue) parts indicate an increase (decrease) in the electron density. The excitation wavelength is indicated in the figure.

is determined to include the electronic interaction region between the $\mathrm{O}_{2}$ molecule and $\mathrm{Ag}$ atoms provided from the calculated electron density differential map in Figs. 2(a) and 2(b) as well as all relevant states that are influenced by $\mathrm{O}_{2}$ adsorption (see also Fig. S4 of the supplementary material). The simulation reveals a significant electron transfer from the Ag surface to the $\mathrm{O}_{2}$ moiety of both orientations (see also Fig. S5 of the supplementary material for the projected DOS), which is consistent with previous calculations using a slab ${ }^{36,37}$ and a cluster model. ${ }^{38,39}$ As a consequence, the $\pi^{*}$ orbital of $\mathrm{O}_{2}$ is partially filled, resulting in a slight elongation of the $\mathrm{O}-\mathrm{O}$ bond $(\sim 0.2 \AA)$. Figures $2(\mathrm{c})$ and $2(\mathrm{~d})$ show the simulated absorption spectrum (colored curves) of the $\mathrm{O}_{2} / \mathrm{Ag}(110)$ cluster using TDDFT calculations. Here, we adopt electronic transition between the ground and excited states via the single-photon process as confirmed in experiment. The simulated spectra reveal characteristic absorptions in the visible and the near-infrared range. In order to obtain the absorption spectra, the oscillator strength (black bars) is broadened by a Lorentzian function. The broadening factor is an arbitrary parameter, and we examine three different widths (full width at half maximum) of 50,100, and $150 \mathrm{~nm}$. We deduced these values from excited-state lifetimes of $10^{-15}$ to $10^{-14} \mathrm{~s}$ which are typically found in adsorbates on metal surfaces, ${ }^{1}$ but further quantitative discussion is inappropriate at the level of the current simulations. In the simulated spectra, there are three absorption bands around 900, 700, and $480 \mathrm{~nm}$.

The direct electron transition at these wavelengths is displayed in Figs. 2(e) $-2(j)$ and listed in Table I. We found that for [001] $-\mathrm{O}_{2}$, electron transfer occurs from the Ag substrate to the hybridized $\pi^{*}$ orbitals of $\mathrm{O}_{2}$ at $708 \mathrm{~nm}$ [the yellow lobes around $\mathrm{O}_{2}$ in Fig. $\left.2(\mathrm{~g})\right]$. This electron transfer causes the elongation of the $\mathrm{O}-\mathrm{O}$ bond and could lead to dissociation. In the case for [110]- $\mathrm{O}_{2}$ at $690 \mathrm{~nm}$, the electron transfer occurs from the occupied to unoccupied states within the hybridized $\pi^{*}$ orbital of $\mathrm{O}_{2}$ [Fig. 2(h)]. Although the contribution of this electron transition to the dissociation is unclear, photo-irradiation may also induce rotation of $\mathrm{O}_{2}$ from the [110]- and [001]-orientation through hotcarrier generation, ${ }^{34}$ promoting the dissociation in the [001] orientation at $\sim 700 \mathrm{~nm}$. In the other cases at 480 and $900 \mathrm{~nm}$, electron transfer takes place from the partially occupied $\pi^{*}$ orbitals to the Ag substrate [Figs. 2(e), 2(f), 2(i), and 2(j); see also the 
TABLE I. Electron transition under several wavelengths from TD-DFT.

\begin{tabular}{lcc}
\hline \hline $\mathrm{O}_{2}$ species & $\lambda_{\text {abs }}(\mathrm{nm})$ & \multicolumn{1}{c}{ Electron transition } \\
\hline$[001]-\mathrm{O}_{2}$ & 926 & $\mathrm{O}_{2} \pi^{*}$ orbitals $\rightarrow \mathrm{Ag}$ substrate \\
& 708 & $\mathrm{Ag}$ substrate $\rightarrow \mathrm{O}_{2} \pi^{*}$ orbitals \\
& 479 & $\mathrm{O}_{2} \pi^{*}$ orbitals $\rightarrow \mathrm{Ag}$ substrate \\
\hline$[1 \overline{1} 0]-\mathrm{O}_{2}$ & 885 & $\mathrm{O}_{2} \pi^{*}$ orbitals $\rightarrow \mathrm{Ag}$ substrate \\
& 690 & $\mathrm{O}_{2} \pi^{*}$ orbitals $\rightarrow \mathrm{O}_{2} \pi^{*}$ orbitals \\
& 476 & $\mathrm{O}_{2} \pi^{*}$ orbitals $\rightarrow$ Ag substrate \\
\hline
\end{tabular}

supplementary material for details]. This transfer compresses the $\mathrm{O}-\mathrm{O}$ bond and then could excite the O-O stretching after relaxation, which could eventually promote dissociation in the ground state. Thus, all the three absorption bands could induce photolysis through the direct charge transfer process. However, the indirect process cannot be ruled out because hot carriers are generated in $\mathrm{Ag}$ through optical absorption in the visible range (5\%-14\%).

Regarding the incident-light-polarization dependence, in the indirect process, p-polarization is more efficient than spolarization. ${ }^{20}$ On the other hand, in the direct process, the above statement is also true considering the transition dipole moment is perpendicular to the surface. Therefore, either mechanism could explain the higher cross section for p-polarization than spolarization.

We now turn to the near-field $\mathrm{O}_{2}$ photolysis in plasmonic STM junctions. Figures 3(a) and 3(b) display the STM image before and after 530-nm illumination with an Ag tip kept in the tunneling regime at the yellow dot position in Fig. 3(a). The incident beam was polarized along the tip axis (p-polarization). After illumination with a relatively low fluence $\left(2.3 \times 10^{17}\right.$ photons $\mathrm{cm}^{-2}$, $2 \mathrm{~mW} \mathrm{~cm}^{-2}$ for $44 \mathrm{~s}$ ), some of the $\mathrm{O}_{2}$ molecules are already dissociated near the tip position [Fig. 3(b)], indicating promotion of the reaction through surface plasmon excitation in the junction. ${ }^{15,16}$ The dissociation cross section depends on the lateral distance from the tip position. Here, we analyze the cross section by dividing the area into two regions as indicated by the dashed lines in Fig. 3(a). The radius of the regions is determined arbitrarily because our purpose is to show the rate difference in molecules at different distances from the tip position. By applying the same counting method as before, the fraction of unreacted $\mathrm{O}_{2}\left(\mathrm{~N}_{\mathrm{O}_{2}} / \mathrm{N}_{\mathrm{i}}\right)$ in both regions 1 and 2 is measured as a function of photon fluence [Fig. 3(c)]. By applying Eq. (1), we obtained the nearfield cross section $\left(\sigma_{\text {diss/NF }}\right)$ of $1.1 \times 10^{-17} \mathrm{~cm}^{2}$ in region 1 and
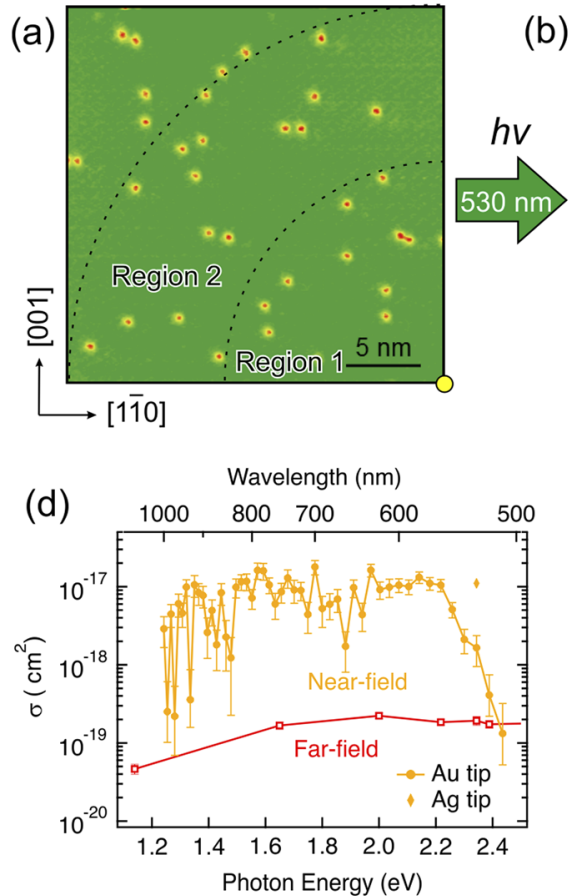
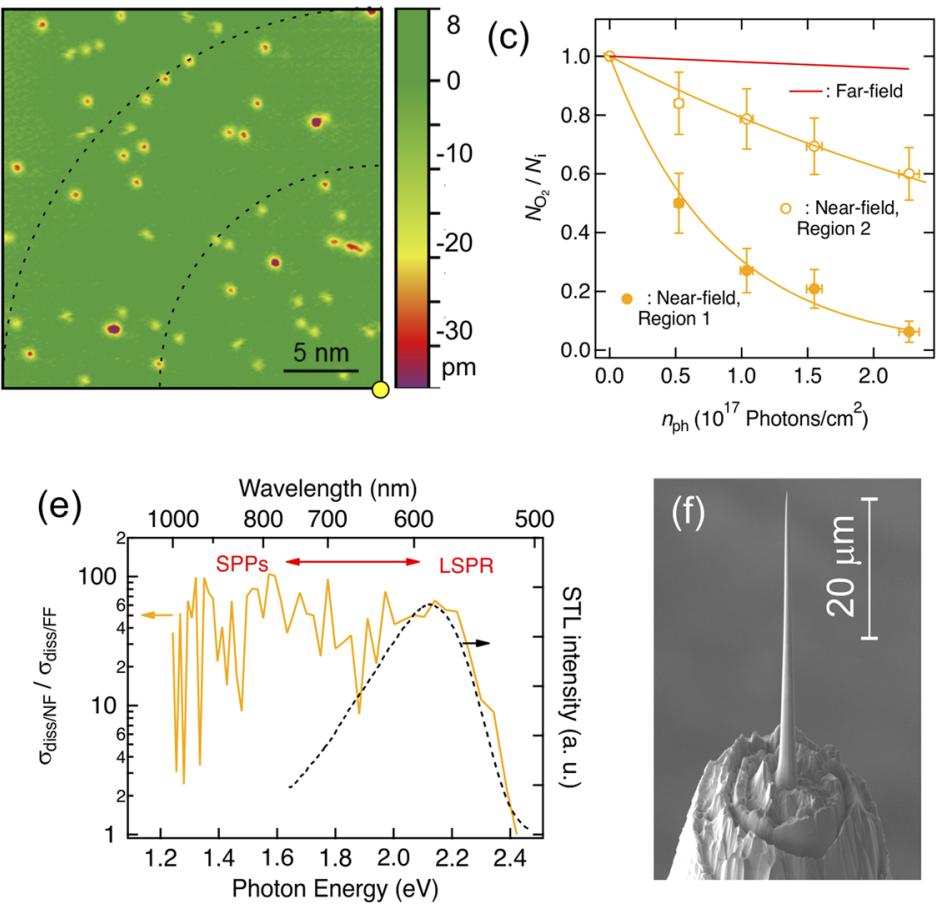

FIG. 3. Near-field photolysis of $\mathrm{O}_{2}$ on $\mathrm{Ag}(110)$. [(a) and (b)] STM images of $\mathrm{O}_{2}$ on $\mathrm{Ag}(110)$ observed at $78 \mathrm{~K}\left(\mathrm{~V}_{\mathrm{s}}=100 \mathrm{mV}\right.$ and $\left.I_{\mathrm{t}}=20 \mathrm{pA}\right)$, (a) before and (b) after illumination with p-polarized light at $530 \mathrm{~nm}\left(2.3 \times 10^{17}\right.$ photons cm $\left.\mathrm{cm}^{-2}\right)$. An Ag tip was kept in the tunneling range $\left(V_{\mathrm{s}}=100 \mathrm{mV}\right.$ and $\left.l_{\mathrm{t}}=20 \mathrm{pA}\right)$ at the bottom right edge of the image frame during illumination. (c) Fraction of unreacted $\mathrm{O}_{2}\left(\mathrm{~N}_{\mathrm{O}_{2}} / \mathrm{N}_{\mathrm{i}}\right)$ as a function of photon fluence at $530 \mathrm{~nm}$ in the two regions shown in (a). Orange curves are the best fitted result of the data points to Eq. (1). (d) Incident photon energy (wavelength) dependence of the near-field photolysis cross section (orange markers) measured in region 1 shown in (a). The cross section by the Ag tip shown in (c) is also marked by diamond. (e) Photolysis enhancement factor $\left(\sigma_{\text {diss } / \mathrm{NF}} / \sigma_{\text {diss/FF }}\right)$ as a function of incident photon energy (wavelength) (orange) and scanning tunneling luminescence spectrum (black dashed line) measured over the Ag(111) surface with $V_{s}=3 V$ and $I_{t}=9 \mathrm{nA}$. (f) $S$ canning electron micrograph of an FIB-milled Au tip. 
$2.2 \times 10^{-18} \mathrm{~cm}^{2}$ in region 2 , revealing 54 - and 10 -fold enhancement with respect to the far-field cross section at $530 \mathrm{~nm}$ [red curve in Fig. 3(c) and Fig. S2 of the supplementary material]. The higher cross section in the region closer to the tip corroborates that the photolysis is associated with local field enhancement in the junction. ${ }^{15,16}$

Figures 3(d) and 3(e) show the wavelength-dependent cross section of the near-field induced photolysis in region 1 [shown in Fig. $3(\mathrm{a})]$ and the photolysis enhancement factor $\left(\sigma_{\text {diss } / \mathrm{NF}} / \sigma_{\text {diss } / \mathrm{FF}}\right)$, respectively. ${ }^{40}$ The data are obtained with an FIB-milled Au tip which has a 50- $\mu \mathrm{m}$-long "polished" shaft, as shown in Fig. 3(f). The cross section is largely enhanced in the red and the near-infrared range. The surface plasmon excitation in the junction promotes the direct charge transfer in three absorption bands (Fig. 2 and Table I) as well as the indirect process (in the latter, hot carriers are generated in the Ag substrate and tip via Landau damping of surface plasmons). The decrease in the enhancement for the Au tip above $2.2 \mathrm{eV}(\lambda<560 \mathrm{~nm})$ results from plasmon quenching due to the interband transition. ${ }^{15}$ On the other hand, when we used an Ag tip, 54-fold enhancement is achieved at $530 \mathrm{~nm}$ [Figs. 3(c) and 3(d)], which is significantly higher than that for the Au tip (8-fold). This can be explained by the different cutoff of surface plasmon excitation resulting from the interband transition. Since the $d$-band edge of $\mathrm{Ag}$ (about $4 \mathrm{eV}$ below the Fermi surface) is much deeper than that of $\mathrm{Au}$ (about $2 \mathrm{eV}),{ }^{41}$ the cutoff of surface plasmon excitation for $\mathrm{Ag}$ is significantly blue-shifted. In other words, an Ag tip can couple with 530-nm light much more efficiently than an Au tip. It should also be noted that local field enhancement may be larger for Ag tips than that for Au tips due to the significantly longer plasmon dephasing time of Ag.

The wavelength-dependent cross section of the $\mathrm{O}_{2}$ photolysis [near-field action spectrum, Fig. 3(d)] exhibits two characteristic regimes: a continuous feature at $500-600 \mathrm{~nm}$ and strong fluctuation at 600-1000 nm. A similar spectral fingerprint has been observed for other near-field action spectra measured with FIB-milled tips. ${ }^{19}$ As discussed in our previous report, the continuous feature (500$600 \mathrm{~nm}$ ) can be attributed to the LSPR in the junction, which is corroborated by the agreement of the spectral response between the scanning tunneling luminescence (STL) and the enhancement factor in Fig. 3(e). On the other hand, the characteristic fluctuation is explained by complex interference and propagation of surface plasmon polaritons (SPPs) generated on the unpolished tip shaft which has submicrometer roughness [as seen in Fig. 3(f)], providing our experimental setup where the whole tip is illuminated by a uniform and spatially extended beam. ${ }^{19,42-45}$ We propose that the surface roughness on the unpolished tip shaft acts as a coupler between the incident light and the SPP (see Fig. S3 and the related calculation in the supplementary material). The launched SPPs then propagate along the smooth milled shaft and focus at the apex. ${ }^{46}$ The contribution of the SPPs becomes more pronounced at a longer wavelength due to the larger propagation distance. The contribution from the propagating SPP results in photolysis in the near-infrared range with the cross section as high as that in the visible range; nevertheless, the intensity of the directly excited LSPR in the junction decreases in the near-infrared range [Fig. 3(e)]. This is a clear contrast to far-field photolysis and is of great importance toward efficient use of sunlight because near infrared makes up $~ 50 \%$ of its total energy on the earth surface.

\section{CONCLUSION}

In summary, we demonstrated that the $\mathrm{O}_{2}$ photolysis on the $\mathrm{Ag}(110)$ surface can be induced by visible light, and the process is observed in real-space using a low-temperature STM. The photolysis mechanism was examined by TD-DFT calculations, revealing optical absorption of the $\mathrm{O}_{2} / \mathrm{Ag}(110)$ complex in the visible and near-infrared range, which promotes photolysis. We propose that the $\mathrm{O}_{2}$ photolysis involves the direct charge transfer process. It was also shown that the photolysis cross section can be largely enhanced in a plasmonic STM junction in both visible and nearinfrared ranges. Our approach paves the way to gain in-depth insight into plasmon-induced photochemistry at the single-molecule level, which will contribute an optical design of plasmonic catalysts and visible- and near-infrared-light driven chemistry.

\section{SUPPLEMENTARY MATERIAL}

The supplementary material includes incident photon flux dependence of the $\mathrm{O}_{2}$ photolysis rate on $\mathrm{Ag}(110)$, far-field photolysis at $530 \mathrm{~nm}$, coupling of incident light to SPPs on the tip shaft by surface roughness, optimized structure for $\mathrm{O}_{2}$ on the $\mathrm{Ag}(110)$ slab, projected density of states of $\mathrm{O}_{2} / \mathrm{Ag}(110)$, and analysis of orbital contribution in the simulated absorption spectra.

\section{ACKNOWLEDGMENTS}

The authors thank A. Hammud for providing FIB tips. This work was supported by KAKENHI Grant Nos. JP16K05725 and JP17H03117 from the Japan Society for the Promotion of Science and the Ministry of Education, Culture, Sports, Science and Technology of Japan (MEXT), the MEXT Projects of "Integrated Research Consortium on Chemical Sciences" and "Elements Strategy Initiative to Form Core Research Center," and JST "Innovative Catalysts," Grant Nos. JPMJCR15P5 and JPMJPR16S6. The computation was mainly carried out using the computer facilities at Research Institute for Information Technology, Kyushu University.

The authors declare no competing financial interest.

\section{REFERENCES}

${ }^{1}$ X.-Y. Zhu, "Surface photochemistry," Annu. Rev. Phys. Chem. 45, 113-144 (1994).

${ }^{2}$ P. Avouris and R. E. Walkup, "Fundamental mechanisms of desorption and fragmentation induced by electronic transitions at surfaces," Annu. Rev. Phys. Chem. 40, 173-206 (1989).

${ }^{3}$ X.-L. Zhou, X.-Y. Zhu, J. M. White, Photochemistry at adsorbate/metal interfaces. Surf. Sci. Rep. 13, 73-220 (1991).

${ }^{4}$ J. T. Yates, Jr. and H. Petek, "Introduction: Photochemistry and photophysics on surfaces," Chem. Rev. 106, 4113-4115 (2006).

${ }^{5}$ L. Hanley, X. Guo, and J. T. Yates, Jr., "Photolysis of chemisorbed dioxygen on Pd(111): Dependence on photon energy," J. Chem. Phys. 91, 7220-7227 (1989).

${ }^{6}$ X.-Y. Zhu, S. R. Hatch, A. Campion, and J. M. White, "Surface photochemistry. II. Wavelength dependences of photoinduced dissociation, desorption, and rearrangement of $\mathrm{O}_{2}$ on $\mathrm{Pt}(111)$," J. Chem. Phys. 91, 5011-5020 (1989).

${ }^{7}$ M. Wolf, E. Hasselbrink, J. M. White, and G. Ertl, "The adsorbate state specific photochemistry of dioxygen on Pd(111)," J. Chem. Phys. 93, 5327-5336 (1990) 
${ }^{8}$ S. R. Hatch, X. Y. Zhu, J. M. White, and A. Campion, "Photoinduced pathways to dissociation and desorption of dioxygen on silver (110) and platinum (111)," J. Phys. Chem. 95, 1759-1768 (1991).

${ }^{9}$ P. Christopher, H. Xin, and S. Linic, "Visible-light-enhanced catalytic oxidation reactions on plasmonic silver nanostructures," Nat. Chem. 3, 467-472 (2011).

${ }^{10} \mathrm{~S}$. Mukherjee et al., "Hot electrons do the impossible: Plasmon-induced dissociation of $\mathrm{H}_{2}$ on Au," Nano Lett. 13, 240-247 (2013).

${ }^{11}$ S. Linic, U. Aslam, C. Boerigter, and M. Morabito, "Photochemical transformations on plasmonic metal nanoparticles," Nat. Mater. 14, 567-576 (2015).

${ }^{12} \mathrm{U}$. Aslam, V. G. Rao, S. Chavez, and S. Linic, "Catalytic conversion of solar to chemical energy on plasmonic metal nanostructures," Nat. Catal. 1, 656-665 (2018).

${ }^{13}$ C. Boerigter, U. Aslam, and S. Linic, "Mechanism of charge transfer from plasmonic nanostructures to chemically attached materials," ACS Nano 10, 61086115 (2016).

${ }^{14}$ C. Boerigter, R. Campana, M. Morabito, and S. Linic, "Evidence and implications of direct charge excitation as the dominant mechanism in plasmon-mediated photocatalysis," Nat. Commun. 7, 10545 (2016).

${ }^{15}$ H. Böckmann, S. Gawinkowski, J. Waluk, M. B. Raschke, M. Wolf, and T. Kumagai, "Near-field enhanced photochemistry of single molecules in a scanning tunneling microscope junction," Nano Lett. 18, 152-157 (2018).

${ }^{16}$ E. Kazuma, J. Jung, H. Ueba, M. Trenary, and Y. Kim, "Real-space and real-time observation of a plasmon-induced chemical reaction of a single molecule," Science 360, 521-526 (2018)

${ }^{17}$ B. K. Hodnett, Heterogeneous Catalytic Oxidation: Fundamental and Technological Aspects of the Selective and Total Oxidation of Organic Compounds (John Wiley, 2000).

${ }^{18}$ Handbook of Heterogeneous Catalysis, edited by G. Ertl, H. Knözinger, F. Schüth, and J. Weitkamp (Wiley-VCH Verlag GmbH \& Co. KGaA, Weinheim, Germany, 2008).

${ }^{19}$ H. Böckmann, M. Müller, A. Hammud, M.-G. Willinger, M. Pszona, J. Waluk, M. Wolf, and T. Kumagai, "Near-field spectral response of optically excited scanning tunneling microscope junctions probed by single-molecule action spectroscopy," J. Phys. Chem. Lett. 10, 2068 (2019).

${ }^{20}$ H. Böckmann, S. Liu, J. Mielke, S. Gawinkowski, J. Waluk, L. Grill, M. Wolf, and T. Kumagai, "Direct observation of photoinduced tautomerization in single molecules at a metal surface," Nano Lett. 16, 1034-1041 (2016).

${ }^{21}$ J. P. Perdew, K. Burke, and M. Ernzerhof, "Generalized gradient approximation made simple," Phys. Rev. Lett. 77, 3865-3868 (1996).

${ }^{22}$ G. Kresse and J. Furthmüller, "Efficiency of ab-initio total energy calculations for metals and semiconductors using a plane-wave basis set," Comput. Mater. Sci. 6, 15-50 (1996).

${ }^{23} \mathrm{G}$. Kresse and J. Furthmüller, "Efficient iterative schemes for $a b$ initio totalenergy calculations using a plane-wave basis set," Phys. Rev. B 54, 11169-11186 (1996).

${ }^{24}$ P. E. Blochl, "Projector augmented-wave method," Phys. Rev. B 50, 1795317979 (1994).

${ }^{25}$ H. J. Monkhorst and J. D. Pack, "Special points for Brillouin-zone integrations," Phys. Rev. B 13, 5188-5192 (1976).

${ }^{26} \mathrm{~K}$. Eichkorn, O. Treutler, H. Öhm, M. Häser, and R. Ahlrichs, "Auxiliary basis sets to approximation Coulomb potentials," Chem. Phys. Lett. 242, 652-660 (1995).

${ }^{27}$ S. Hirata and M. Head-Gordon, "Time-dependent density functional theory within the Tamm-Dancoff approximation," Chem. Phys. Lett. 314, 291-299 (1999).
${ }^{28}$ R. Ahlrichs, M. Bär, M. Häser, H. Horn, and C. Kölmel, "Electronic structure calculations on workstation computers: The program system turbomole," Chem. Phys. Lett. 162, 165-169 (1989).

${ }^{29} \mathrm{~F}$. Weigend and R. Ahlrichs, "Balanced basis sets of split valence, triple zeta valence and quadruple zeta valence quality for $\mathrm{H}$ to $\mathrm{Rn}$ : Design and assessment of accuracy," Phys. Chem. Chem. Phys. 7, 3297-3305 (2005).

${ }^{30} \mathrm{~K}$. Momma and F. Izumi, "VESTA 3 for three-dimensional visualization of crystal, volumetric and morphology data," J. Appl. Crystallogr. 44, 1272-1276 (2011).

${ }^{31}$ N. M. O’Boyle, A. L. Tenderholt, and K. M. Langner, "cclib: A library for package-independent computational chemistry algorithms," J. Comput. Chem. 29, 839-845 (2008).

${ }^{32}$ F. Bartolucci, R. Franchy, J. C. Barnard, and R. E. Palmer, "Two chemisorbed species of $\mathrm{O}_{2}$ on $\mathrm{Ag}(110)$," Phys. Rev. Lett. 80, 5224-5227 (1998).

${ }^{33} \mathrm{~J}$. R. Hahn and W. Ho, "Chemisorption and dissociation of single oxygen molecules on Ag(110)," J. Chem. Phys. 123, 214702 (2005).

${ }^{34}$ J. R. Hahn, S. H. Jang, K. W. Kim, and S. B. Son, "Hot carrier-selective chemical reactions on $\mathrm{Ag}(110)$," J. Chem. Phys. 139, 074707 (2013).

${ }^{35} \mathrm{~F}$. E Olsson, N. Lorente, and M. Persson, "STM images of molecularly and atomically chemisorbed oxygen on silver," Surf. Sci. 522, L27-L35 (2003).

${ }^{36}$ P. A. Gravil, D. M. Bird, and J. A. White, "Adsorption and dissociation of $\mathrm{O}_{2}$ on Ag(110),” Phys. Rev. Lett. 77, 3933-3936 (1996).

${ }^{37}$ T. B. Rawal, S. Hong, A. Pulkkinen, M. Alatalo, and T. S. Rahman, "Adsorption, diffusion, and vibration of oxygen on Ag(110)," Phys. Rev. B 92, 035444 (2015).

${ }^{38}$ P. J. van Den Hoek and E. J. Baerends, "Chemisorption and dissociation of $\mathrm{O}_{2}$, on Ag(110),” Surf. Sci. 221, L791-L799 (1989).

${ }^{39}$ H. Nakatsuji and H. Nakai, "Dipped adcluster model study for molecular and dissociative chemisorptions of $\mathrm{O}_{2}$ on Ag surface," J. Chem. Phys. 98, 2423-2436 (1993).

${ }^{40}$ Due to the experimental endurance (time constraint), the photolysis cross section at each wavelength was calculated only from two data points: the number of initial $\mathrm{O}_{2}\left(\mathrm{~N}_{\mathrm{i}}\right)$ and the number of unreacted $\mathrm{O}_{2}\left(\mathrm{~N}_{\mathrm{O}_{2}}\right)$ after illumination at a fixed fluence of $3.27 \times 10^{16}$ photons $\mathrm{cm}^{-2}$. The $\sigma_{\text {diss } / \mathrm{NF}}$ was determined by $\frac{\sigma_{\text {diss }}}{N F}=\frac{\ln \left(N_{\mathrm{O}_{2}} / N_{\mathrm{i}}\right)}{n_{\text {ph }}}$.

${ }^{41}$ N. V. Smith, "Photoemission spectra and band structures of d-band metals. III. Model band calculations on Rh, Pd, Ag, Ir, Pt, and Au," Phys. Rev. B 9, 1365-1376 (1974).

${ }^{42}$ S. Grésillon, L. Aigouy, A. C. Boccara, J. C. Rivoal, X. Quelin, C. Desmarest, P. Gadenne, V. A. Shubin, A. K. Sarychev, and V. M. Shalaev, "Experimental observation of localized optical excitations in random metal-dielectric films," Phys. Rev. Lett. 82, 4520-4523 (1999).

${ }^{43}$ V. Emiliani, F. Intonti, M. Cazayous, D. S. Wiersma, M. Colocci, F. Aliev, and A. Lagendijk, "Near-field short range correlation in optical waves transmitted through random media," Phys. Rev. Lett. 90, 250801 (2003).

${ }^{44}$ N. Caselli, F. Intonti, F. La China, F. Biccari, F. Riboli, A. Gerardino, L. Li, E. H. Linfield, F. Pagliano, A. Fiore et al., "Near-field speckle imaging of light localization in disordered photonic systems," Appl. Phys. Lett. 110, 081102 (2017). ${ }^{45} \mathrm{~J}$.-J. Greffet and R. Carminati, "Relationship between the near-field speckle pattern and the statistical properties of a surface," Ultramicroscopy 61, 43-50 (1995).

${ }^{46}$ S. Berweger, J. M. Atkin, R. L. Olmon, and M. B. Raschke, "Light on the tip of a needle: Plasmonic nanofocusing for spectroscopy on the nanoscale," J. Phys. Chem. Lett. 3, 945-952 (2012). 